\title{
Lactulose inhibits endotoxin induced tumour necrosis factor production by monocytes. An in vitro study
}

\author{
J W Greve, D J Gouma, P A M v Leeuwen, W A Buurman
}

\begin{abstract}
Preoperative oral treatment with lactulose is used to prevent complications after surgery in patients with obstructive jaundice. The effect is perhaps the result of an inactivation of gut derived endotoxins but the exact mechanism of action is, however, unknown. Tumour necrosis factor is an important mediator of endotoxin toxicity. The cytokine tumour necrosis factor is mainly produced by mononuclear phagocytes. In this study, the effect of lactulose on the endotoxin induced tumour necrosis factor release by monocytes was investigated. The direct effect of lactulose on endotoxin was tested in a chromogenic limulus amoebocyte lysate assay. Polymyxin B a known inactivator of endotoxin was used as control in both experiments. Lactulose has a limited capacity to inactivate endotoxin as measured in the endotoxin assay. In contrast lactulose significantly reduced endotoxin induced tumour necrosis factor production by monocytes. In conclusion lactulose inhibits tumour necrosis factor production by a direct inhibitory effect on monocytes, rather than by inactivation of endotoxin. Because tumour necrosis factor is an important mediator of endotoxin toxicity, this inhibitory effect could explain the beneficial effect of lactulose in obstructive jaundice.
\end{abstract}

Gut derived endotoxins are thought to be responsible for postoperative complications such as renal insufficiency, gastrointestinal bleeding and infections, in patients with obstructive jaundice. ${ }^{12}$ This theory is supported by the high incidence of endotoxaemia reported in these patients and the correlation that was found between the presence of endotoxaemia and occurrence of complications. . $^{3-5}$

Polymyxin B is a known antiendotoxin agent ${ }^{6-8}$ and reduces mortality in rats, with experimental biliary obstruction, challenged with intragastric endotoxin. ${ }^{9}$ Unfortunately, because of its renal toxicity, effective doses cannot be given to jaundiced patients. ${ }^{10}$ Orally administrated bile acids have been used successfully to prevent endotoxin related complications after surgery in jaundiced patients ${ }^{1112}$ bile acids reduced absorption of endotoxins from the gastrointestinal tract resulting in a decrease of portal and systemic endotoxaemia and of postoperative renal complications.

Preoperative oral treatment with lactulose was found to be equally effective in preventing postoperative renal complications in patients with obstructive jaundice. ${ }^{13}$ The effect of lactulose was explained by an inactivation of endotoxins from the gastrointestinal tract. Suggestive evidence for this hypothesis was the reduced limulus amoebocyte lysate activation by endotoxin in the presence of lactulose..$^{13+}$ The mechanism through which lactulose reduces endotoxin toxicity, however, remains to be further clarified.

Tumour necrosis factor is reported to be an important mediator of endotoxin toxicity. ${ }^{15-17}$ The cytokine tumour necrosis factor is mainly produced by mononuclear phagocytes in response to endotoxin. ${ }^{18} 19$ These findings enabled us to develop an in vitro model to study the effect of different agents on the production of tumour necrosis factor as the mediator of endotoxin toxicity. Human peripheral blood monocytes were stimulated with endotoxin in the presence of the compound to be tested and tumour necrosis factor production was measured with an enzyme linked immunosorbent assay (ELISA).

To analyse if and how lactulose prevents the toxic effects of endotoxin, this in vitro model was used. The direct effect of lactulose on endotoxin was evaluated with a modified chromogenic limulus amoebocyte lysate assay. As lactulose preparations used in clinical practice can be divided in two groups, a crystalline pure lactulose and lactulose syrup containing galactose and lactose besides lactulose, both preparations were tested.

\section{Methods}

\section{CHEMICALS AND ANTIBODIES}

Lactulose syrup (Duphalac ${ }^{\circledR}$ ) was kindly provided by Duphar (Weesp, The Netherlands). Crystalline lactulose (Legendal ${ }^{\circledR}$ ) was a gift from Inpharzam (Almere, The Netherlands). Lactitol was kindly provided by CGA biochemicals (Gorichem, The Netherlands). Lipopolysaccharide $E$ coli 0111:B4 chromatographically purified, phorbol myristate acid, Polymyxin B sulphate $(8000 \mathrm{U} / \mathrm{mg})$, latex beads $(1.091 \mu \mathrm{m}$ diameter), bovine serum albumin, and ophenylenediamine were obtained from Sigma Chemical Co (St Louis, MO). Human recombinant tumour necrosis factor was a gift of BASF/ Knoll AG (Ludwigshaven, FRG). Monoclonal antibodies against recombinant tumour necrosis factor were derived from hybridomas obtained by standard cell fusion procedures. Rabbit polyclonal antibodies against tumour necrosis factor were raised by immunising rabbits with recombinant tumour necrosis factor. Peroxidase conjugated goat antirabbit IgG was purchased from 
Jackson Immunoresearch Laboratories (West Grove, PA).

\section{MONONUCLEAR CELLS FROM HUMAN PERIPHERAL BLOOD}

Peripheral blood mononuclear cells from buffy coats of healthy blood donors (kindly provided by the local blood bank) were purified by buoyant density centrifugation on Lymphoprep (Nycomed AS, Oslo, Norway). The interface layer was collected and washed twice in Hanks balanced salt solution. The peripheral blood mononuclear cells were resuspended in culture medium, consisting of RPMI 1640 (Gibco, Paisley, Scotland) supplemented with $10 \%$ bovine calf serum (Hyclone, Logan, UT) and streptomycin $100 \mu \mathrm{g} / \mathrm{ml}$ penicillin $100 \mathrm{IU} / \mathrm{ml}$ (Flow, Irvine, UK), at a concentration of $5 \times 10^{7}$ / $\mathrm{ml}$. Monocytes were further isolated as described elsewhere. ${ }^{20}$ In short, the suspension was spun in polypropylene tubes at $20 \mathrm{rpm}, 4^{\circ} \mathrm{C}$ to induce monocyte clumping. The clumped monocytes were separated from lymphocytes by sedimentation on ice cold bovine calf serum for $20 \mathrm{~min}$. Monocytes were resuspended in culture medium at a concentration of $105 / \mathrm{ml}$ and transferred to a 96 well tissue culture plate (Costar, Cambridge, MA) $100 \mu \mathrm{l} /$ well. The remaining cells consisted of more than $90 \%$ monocytes as evaluated by phagocytosis and indirect immunofluorescence (data not shown).

\section{TUMOUR NECROSIS FACTOR ENZYME}

LINKED IMMUNOSORBENT ASSAY (ELISA)

Tumour necrosis factor concentrations in the cell free supernatants of the cell cultures were determined with a tumour necrosis factor specific ELISA as described previous. ${ }^{21}$ In short: 96 well immuno plates (Nunc, Roskilde, Denmark) were coated with a monoclonal antitumour necrosis factor antibody. Test samples were added to the plate and incubated for three hours at room temperature. A standard titration curve was obtained by making serial dilutions of a known sample of recombinant tumour necrosis factor. Next the plates were washed and sequentially incubated with rabbit antitumour necrosis factor immune serum and peroxidase conjugated goat antirabbit IgG. After adding substrate (o-phenylenediamine $0.43 \mathrm{mg} / \mathrm{ml}$ ) the colour reaction was stopped and light absorption (at 492 $\mathrm{nm}$ ) was measured with a microelisa autoreader (Flow, Irvine, UK). The ELISA has a lower detection limit of $10 \mathrm{pg} / \mathrm{ml}$. The tumour necrosis factor-assay was neither affected by both lactulose preparations nor by Polymyxin B. Extinctions as measured with the ELISA of different concentrations of recombinant tumour necrosis factor as well as natural, monocyte derived, human tumour necrosis factor in presence of these compounds differed less than $5 \%$ from control values.

ENDOTOXIN ASSAY

Endotoxin was measured with a modified chromogenic limulus amoebocyte lysate test (Coates ${ }^{\circledR}$ endotoxin, Kabivitrum, Stockholm,
Sweden). ${ }^{22} 23$ To evaluate the direct inactivating effect of lactulose on endotoxin crystalline lactulose or lactulose syrup dissolved in pyrogen free water (NPBI, Emmer-Compascuum, The Netherlands), at concentrations similar to those used in the cell culture experiments, were incubated for two hours at $37^{\circ} \mathrm{C}$ with different concentrations of lipopolysaccharide. As control Polymyxin B, a known inactivator of endotoxin, was incubated at a concentration of $25 \mu \mathrm{g} / \mathrm{ml}$ with lipopolysaccharide. After incubation the remaining endotoxin was measured with the limulus amoebocyte lysate assay. All glassware used in the experiments was rendered endotoxin free by heating for three hours at $180^{\circ} \mathrm{C}$. Addition of lactulose or Polymyxin B just before the limulus lysate or the substrate step of the limulus amoebocyte lysate assay did not affect recovery of standard samples of lipopolysaccharide. This indicates that both the lysate reaction and the substrate activation are not influenced by both substances.

\section{CELL CULTURE EXPERIMENTS}

The isolated monocytes, as described above, were allowed to settle for two hours after which culture medium was replaced with culture medium to which combinations of lipopolysaccharide, phorbol myristate acid, crystalline lactulose, lactulose syrup, lactitol, and Polymyxin B were added. In the first series of experiments a serial dilution of lipopolysaccharide was preincubated for two hours with crystalline lactulose, lactulose syrup, lactitol, or Polymyxin B. In the second series of experiments different concentrations of crystalline lactulose, lactulose syrup, and Polymyxin B were preincubated for two hours with a serial dilution of lipopolysaccharide. In the third series of experiments phorbol myristate acid was used to stimulate the tumour necrosis factor production of monocytes, again after preincubation for two hours with crystalling lactulose, lactulose syrup, lactitol, and Polymyxin B. In both experiments monocytes stimulated with lipopolysaccharide or phorbol myristate acid in culture medium served as positive controls whereas monocytes cultured in culture medium were used as negative controls. All cultures were performed at $37^{\circ} \mathrm{C}$ in a moist $8 \%$ carbon dioxide air atmosphere. Lactulose syrup, crystalline lactulose, and lactitol used in the cell culture experiments were diluted in pyrogen free water in order to reach an osomolarity of 300 mosmol. Thereafter lactulose syrup was diluted in culture medium to obtain a concentration of $\pm 7 \mathrm{mg} / \mathrm{ml}$ of lactulose. Crystalline lactulose and lactitol were diluted in culture medium to a concentration of $\pm 10 \mathrm{mg}$ / $\mathrm{ml}$. The difference in lactulose content between the two lactulose solutions is caused by the presence of the monosaccharides lactose and galactose which account for $30 \%$ of the osmolarity of undiluted lactulose syrup. In all experiments osmolarity and $\mathrm{pH}$ of culture medium after addition of the different compounds were determined. Osmolarity ranged from 300 to 320 mosmol, $\mathrm{pH}$ from $7 \cdot 14$ to 7.24 . The values of culture medium were 307 mosmol and $\mathrm{pH} 7 \cdot 15$. 
TABLE Effect of lactulose and Polymyxin B on endotoxin measured with the limulus amoebocyte lysate assay

\begin{tabular}{lcll}
\hline & $A$ & $B$ & $C$ \\
\hline Control & $7 \cdot 6(8 \cdot 1)$ & $43 \cdot 3(14 \cdot 0)$ & $165(18 \cdot 8)$ \\
Crystalline lactulose & $20 \cdot 2(13 \cdot 4)$ & $72 \cdot 3(8 \cdot 6)$ & $149 \cdot 8(20 \cdot 9)$ \\
Lactulose syrup & $2 \cdot 3(4 \cdot 8)$ & $14 \cdot 5(5 \cdot 8)$ & $85(6 \cdot 5)$ \\
Polymyxin B & $1 \cdot 8(2 \cdot 1)$ & $6 \cdot 7(5 \cdot 9)$ & $11 \cdot 2(7 \cdot 9)$ \\
\hline
\end{tabular}

Endotoxins (in three concentrations A, B, C), dissolved in endotoxin free water, were incubated with lactulose syrup $(7 \mathrm{mg}$ lactulose $/ \mathrm{ml}$ ), crystalline lactulose ( $10 \mathrm{mg}$ lactulose $/ \mathrm{ml})$, and Polymyxin B $(25 \mu \mathrm{g} / \mathrm{ml})$ for two hours at $37^{\circ} \mathrm{C}$. Untreated lipopolysaccharide dissolved in endotoxin free water served as control. Remaining endotoxin was measured with a modified chromogenic limulus amoebocyte lysate assay. Results, expressed in pg lipopolysaccharide/ml, are mean (SD) of 6 values.

Experiments lasted for 16-18 hours after which cell free culture supernatants were harvested for analysis. The supernatants were assayed for presence of tumour necrosis factor directly or were stored at $-20^{\circ} \mathrm{C}$ until analysis. Experiments were performed in duplicate or quadruplicate as given below. All experiments were repeated at least three times using monocytes from different blood donors.

\section{VIABILITY OF MONOCYTES}

Viability of the monocytes was examined after the experiments by trypan blue exclusion and by phagocytosis of latex beads. Latex beads were washed four times in phosphate buffered saline and a $0.01 \%(\mathrm{v} / \mathrm{v})$ suspension was made in culture medium. The latex beads were added to the monocytes. After incubation for two hours at $37^{\circ} \mathrm{C}$ remaining latex beads were removed and percentage phagocytosis was assessed by counting the monocytes containing two or more latex beads. The percentage trypan blue excluding, viable, cells was assessed by counting 200 cells per cell culture.

\section{Results}

EFFECT OF LACTULOSE AND POLYMYXIN B ON LIMULUS AMOEBOCYTE LYSATE ACTIVATION BY ENDOTOXIN

The concentrations of both lactulose prepararepresent mean $(S D)$ of 4 values. tions used to inactivate endotoxin were similar to the concentrations used in the cell culture experiments. Lactulose syrup (Duphalac) inactivated low amounts of lipopolysaccharide significantly, however at increasing amounts of lipopolysaccharide this inactivating effect of lactulose decreased, being reduced to approximately $50 \%$ at an endotoxin concentration of $150 \mathrm{pg} / \mathrm{ml}$ (Table). The crystalline, $99 \%$ pure, lactulose (Legendal) did not inactivate lipopolysaccharide as measured with the limulus amoebocyte lysate assay.

As a positive control Polymyxin B, a known inactivator of lipopolysaccharide, was used. In contrast with both lactulose preparations Polymyxin B inactivated the amount of lipopolysaccharide used in this experiment nearly completely.

EFFECT OF LACTULOSE, LACTITOL, AND POLYMYXIN B ON THE TUMOUR NECROSIS FACTOR PRODUCTION BY MONOCYTES STIMULATED WITH ENDOTOXIN Stimulation of monocytes with endotoxin resulted in a high tumour necrosis factor release. Endotoxin dosages of $1 \mathrm{ng}$ lipopolysaccharide/ml caused tumour necrosis factor productions which were on average above $1000 \mathrm{pg} / \mathrm{ml}$ (Figs 1-3). The tumour necrosis factor production rapidly increased at higher lipopolysaccharide dosages reaching a plateau at 30-100 ng lipopolysaccharide/ml, after which a much slower increase of tumour necrosis factor production in relation to the lipopolysaccharide dose was found. Measuring tumour necrosis factor production by monocytes was a highly sensitive and reproducible way to detect the presence of biological active lipopolysaccharide.

Both lactulose preparations inhibited tumour necrosis factor production by monocytes after stimulation with endotoxin significantly (Fig 1). Lactulose syrup appeared to be a more effective inhibitor of tumour necrosis factor production, than crystalline lactulose. At $7 \mathrm{mg}$ lactulose $/ \mathrm{ml}$ lactulose syrup reduced lipopolysaccharide induced tumour necrosis factor production by monocytes with $95 \%$ at all concentrations of lipopolysaccharide used, whereas crystalline lactulose in similar concentrations reduced tumour necrosis factor production with $15-20 \%$. Lactitol at $10 \mathrm{mg} / \mathrm{ml}$ did not inhibit endotoxin induced tumour necrosis factor production by monocytes (data not shown in figure).

As expected Polymyxin $B$, a proven inactivator of endotoxins, inhibited tumour necrosis factor production by monocytes after stimulation with endotoxins. With $25 \mu \mathrm{g}$ Polymyxin $\mathrm{B} / \mathrm{ml}$ on average $30 \mathrm{ng}$ lipopolysaccharide/ml was inactivated.

The effect of both lactulose preparations and Polymyxin B on the endotoxin induced tumour necrosis factor production by monocytes was further studied by testing different concentrations of these agents. Crystalline lactulose and lactulose syrup inhibited concentration dependent lipopolysaccharide induced tumour necrosis factor production by monocytes (Figs 2, 3). Lactulose syrup reduced lipopolysaccharide induced tumour necrosis factor production at 7 , 


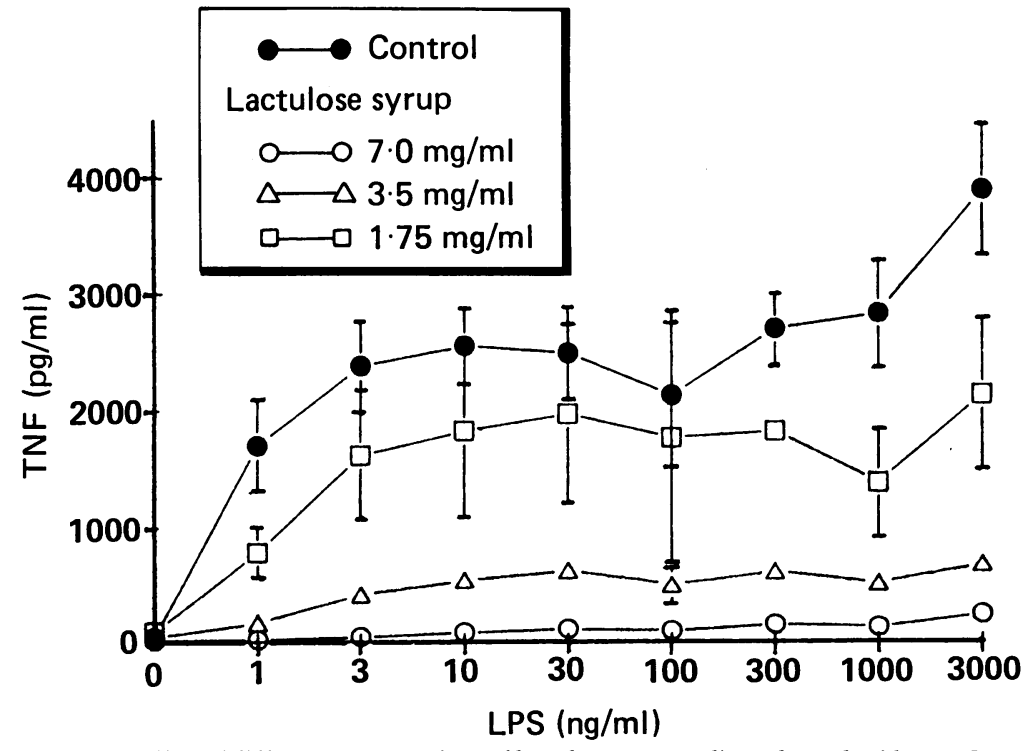

Figure 2: Effect of different concentrations of lactulose syrup on lipopolysaccharide (LPS) induced tumour necrosis factor (TNF) production by monocytes. Monocytes were incubated with a serial dilution of lipopolysaccharide preincubated with lactulose syrup in concentrations of $7,3 \cdot 5$, and $1 \cdot 75 \mathrm{mg}$ lactulose $\mathrm{ml}$. Untreated lipopolysaccharide served as control. Tumour necrosis factor production was measured in cell free culture supernatants with an ELISA. Data Points represent mean (SD) of 4 values.

3.5 , and $1.75 \mathrm{mg}$ lactulose $/ \mathrm{ml}$ with 95,75 , and $30 \%$ respectively. Crystalline lactulose reduced lipopolysaccharide induced tumour necrosis factor production with approximately 25,15 , and $0 \%$ at concentrations of 10,5 , and $2.5 \mathrm{mg}$ lactulose/ml. This inhibitory effect of both lactulose preparations was independent from the concentration of endotoxin used. Polymyxin B also reduced concentration dependent the tumour necrosis factor production of monocytes stimulated with endotoxin. With concentrations of 25,5 , and $1 \mu \mathrm{g}$ Polymyxin $\mathrm{B} / \mathrm{ml}$ the effect of 30,10 , and $1 \mathrm{ng}$ lipopolysaccharide/ml was inhibited. In contrast with lactulose, however, this inhibitory effect was limited to a maximum

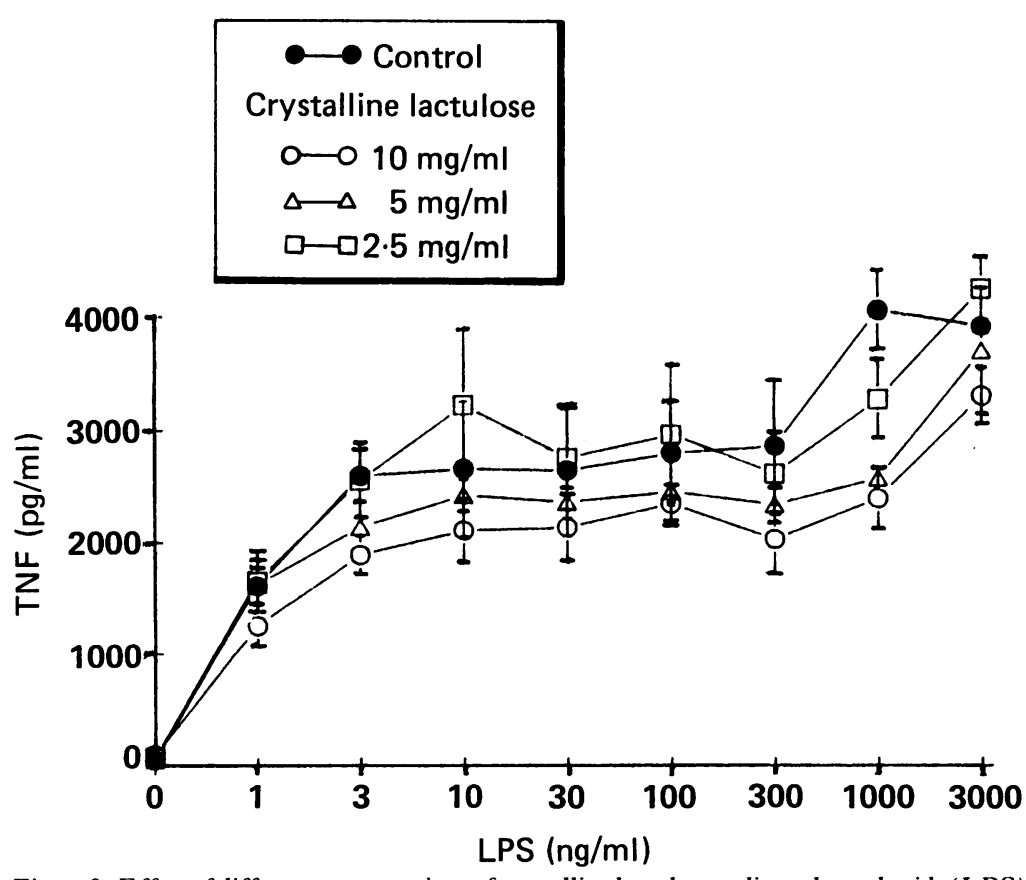

Figure 3: Effect of different concentrations of crystalline lactulose on lipopolysaccharide (LPS induced tumour necrosis factor $(T N F)$ production by monocytes. Monocytes were incubated with a serial dilution of LPS preincubated with crystalline lactulose in concentrations of 10,5 , and $2.5 \mathrm{mg}$ lactulose/ml. Tumour necrosis factor production was measured in cell free culture supernatants with an ELISA. Data points represent mean $(S D)$ of 4 values. dose of endotoxin, after which tumour necrosis factor production of Polymyxin B treated cells paralleled that of control monocytes (Fig 4).

TUMOUR NECROSIS FACTOR PRODUCTION BY MONOCYTES AFTER STIMULATION WITH PHORBOL MYRISTATE ACID, EFFECT OF LACTULOSE, LACTITOL AND POLYMYXIN B

To further analyse the effect of both lactulose preparations, lactitol, and Polymyxin B, on tumour necrosis factor production by monocytes, the phorbol myristate acid induced tumour necrosis factor production by monocytes was studied. Phorbol myristate acid induces tumour necrosis factor production by an activation of the intracellular messenger protein kinase $\mathrm{C}$.

After stimulation with phorbol myristate acid monocytes produced high amounts of tumour necrosis factor. Crystalline lactulose and lactulose syrup in concentrations shown, significantly reduced phorbol myristate acid induced tumour necrosis factor production by monocytes (Fig 5). This reduction was equivalent to the inhibitory effect of both crystalline lactulose and lactulose syrup on endotoxin induced tumour necrosis factor production by monocytes. Lactitol did not inhibit phorbol myristate acid induced tumour necrosis factor production.

Polymyxin B did not affect phorbol myristate acid induced tumour necrosis factor production by monocytes, this in contrast to the endotoxin induced tumour necrosis factor production.

VIABILITY OF THE MONOCYTES AFTER THE EXPERIMENTS

After the cell culture experiments the viability of the monocytes was tested by trypan blue exclusion and phagocytosis of latex beads. Directly after removing supernatants for tumour necrosis factor measurements trypan blue solution or latex beads in culture medium were added to the cell cultures. Trypan blue exclusion was greater than $85 \%$ in all experiments. Phagocytosis of latex beads was clearly delayed, but not significantly reduced, after incubation with lactulose syrup whereas preincubation with crystalline lactulose or Polymyxin B did not affect phagocytosis as compared with control cells.

\section{Discussion}

The results of our experiments show that crystalline lactulose (Legendal) did not inactivate endotoxin as determined with the limulus amoebocyte lysate assay. Lactulose syrup (Duphalac) did inactivate low amounts of lipopolysaccharide, this effect decreased with increasing lipopolysaccharide concentrations.

Both lactulose syrup and crystalline lactulose inhibited tumour necrosis factor production of monocytes stimulated with lipopolysaccharide significantly. Polymyxin B also inhibited tumour necrosis factor production by monocytes after stimulation with endotoxin but the kinetics were 


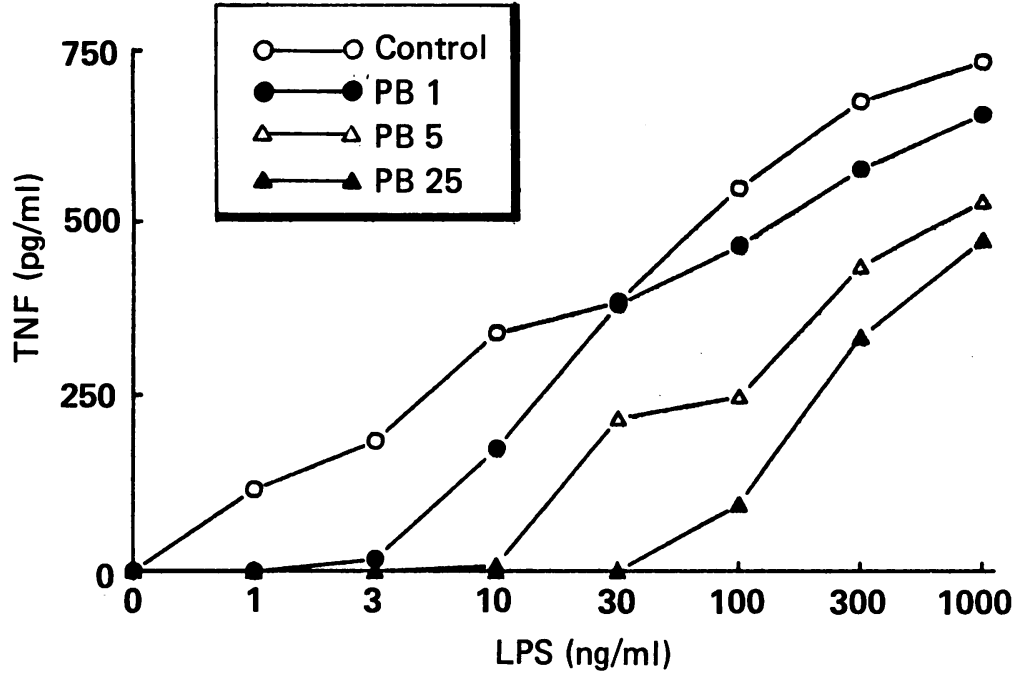

Figure 4: Effect of different concentrations of Polymyxin B on lipopolysaccharide (LPS) induced tumour necrosis factor $(T N F)$ production by monocytes. Monocytes were incubated with a serial dilution of lipopolysaccharide preincubated with Polymyxin B in concentrations of 25,5 , and $1 \mu \mathrm{g} / \mathrm{ml}$. Tumour necrosis factor production was measured in cell free culture supernatants with an ELISA. Results from a representative experiment are shown.

clearly different from the inhibition caused by lactulose. Tumour necrosis factor production of the monocytes stimulated with Polymyxin B treated lipopolysaccharide parallelled that of control cells when a maximum amount of lipopolysaccharide was passed. Apparently Polymyxin B could only inactivate a certain amount of endotoxin related to the amount of Polymyxin B used. In contrast both lactulose preparations inhibited tumour necrosis factor production by monocytes independent from the concentration of endotoxin used. These data indicate that where Polymyxin B was effective by actually inactivating endotoxin, both lactulose preparations probably prevented the production of the mediator of endotoxin toxicity by a direct effect on the cells. The inhibitory effect of both lactulose preparations or Polymyxin B in the experiments could not be explained by a cytolytic effect on the cells as the viability of

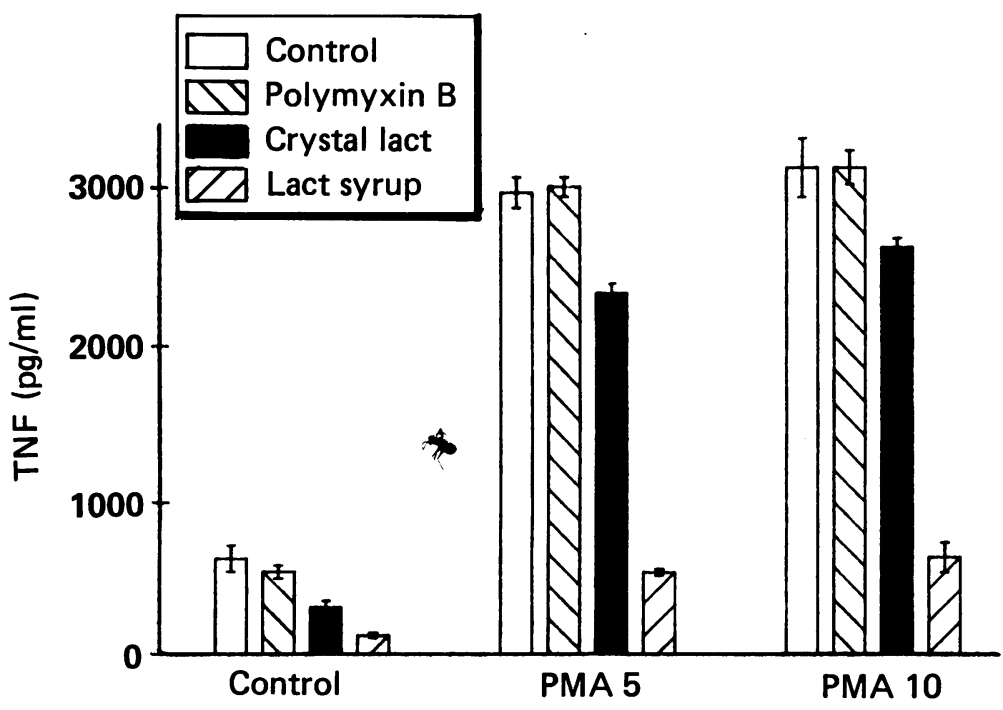

Figure 5: Effect of both lactulose preparations and Polymyxin B on the phorbol myristate acid (PMA) induced tumour necrosis factor production by monocytes. Monocytes were incubated with PMA in concentrations of 5 and $10 \mathrm{ng} / \mathrm{ml}$ in the presence of lactulose syrup ( $7 \mathrm{mg}$ lactulose $\mathrm{ml}$ ), crystalline lactulose (10 mg lactulose/ml), and Polymyxin B $25 \mu \mathrm{g} / \mathrm{ml}$. Unstimulated monocytes and monocytes stimulated with PMA served as control. Bars represent mean $(S D)$ of 6 values. monocytes was not affected. Lactitol a disaccharide clinically used for similar indications as lactulose did not reduce tumour necrosis factor production by monocytes, indicating that the observed inhibitory effect is specific for lactulose.

Experiments with phorbol myristate acid, a direct stimulator of tumour necrosis factor production by monocytes which acts through activation of the intracellular messenger protein kinase $\mathrm{C}$, supplied further evidence for a direct inhibitor effect of lactulose on monocytes. Both lactulose preparations inhibited the phorbol myristate acid induced tumour necrosis factor production by monocytes in a fashion similar to the inhibition of lipopolysaccharide induced tumour necrosis factor production. In contrast Polymyxin B, as expected, did not reduce tumour necrosis factor production by monocytes stimulated with phorbol myristate acid.

The data show that both lactulose preparations inhibit lipopolysaccharide induced as well as phorbol myristate acid induced tumour necrosis factor production by monocytes. In all cell culture experiments, however, lactulose syrup was more effective than similar amounts of crystalline lactulose in reducing tumour necrosis factor production by monocytes.

The effect of Polymyxin B as described in our experiments is similar to results of a study by Duff et al. ${ }^{24}$ In these experiments the effect of Polymyxin B on production of endogenous pyrogen by monocytes after stimulation with endotoxin was studied. They found that lipopolysaccharide induced production of endogenous pyrogen was inhibited by Polymyxin B, whereas production of endogenous pyrogen after stimulation of monocytes with killed staphylococci could not be reduced by polymyxin $B$. This indicated that Polymyxin B inactivated lipopolysaccharide but did not affect the monocytes. The inhibitory effect of Polymyxin B on lipopolysaccharide but not on cells was further supported by results of Spear et al, who used Polymyxin B to inactivate contaminating lipopolysaccharide in mixed lymphocyte reactions and found that Polymyxin B did not affect $\left[{ }^{3} \mathrm{H}\right]$ thymidine incorporation. ${ }^{25}$ Polymyxin B was used successfully as an antiendotoxin drug in a number of in vivo experiments, but its high toxicity severely limits its clinical use..$^{6-10}$ In contrast with Polymyxin B little is known of the antiendotoxin effect of lactulose.

Orally administrated lactulose prevented endotoxin related complications after surgery in jaundiced patients. ${ }^{13}$ Moreover galactosamine induced liver necrosis, caused by endotoxins from the gastrointestinal tract, was prevented by oral pretreatment with lactulose. ${ }^{1+}$ Both studies reported a strongly reduced activation of the limulus amoebocyte lysate assay by endotoxin in presence of lactulose but these data differ from our observations. We found that lactulose inactivates only small amounts of endotoxin. The results of our study indicate, however, that the efficacy of lactulose can be explained by a direct effect on mononuclear phagocytes resulting in a reduced production of the mediator of endotoxin toxicity. Lehman et al showed that tumour necrosis factor appeared to be a central 
mediator of endotoxin mediated mortality in mice treated with $\mathrm{D}$-galactosamine and endotoxin. ${ }^{19}$ These results indicate that inhibition of tumour necrosis factor production can prevent endotoxin induced complications.

That lactulose treatment not only causes an acidification and/or hyperosmolarity of the bowel lumen, but can also have an effect on metabolism of bacteria and different cells in the bowel wall was suggested by a number of studies on lactulose treatment of hepatic encephalopathy in patients with liver cirrhosis. Diminished ammonia production after lactulose treatment was explained by alteration of bacterial flora in the gut, by changes of bacterial metabolism, and recently by an effect of lactulose on metabolism of crypt and villus cells of the bowel wall. ${ }^{26-30}$

Lactulose under normal conditions is only absorbed in small amounts from the gastrointestinal tract (approximately $1.5 \%$ of the administrated oral dose is excreted in urine).${ }^{31}$ In situations of mucosal damage, stress, or infection, however, the absorption of lactulose from the bowel is greatly increased. ${ }^{32-34}$ It is in these situations where substantial amounts of lactulose penetrate the bowel wall that it is probably most effective. Our observations show that lactulose can have a direct effect on cell function. Lactulose inhibited the release of tumour necrosis factor, the mediator of endotoxin toxicity, by an inhibitory effect on mononuclear phagocytes. The effect of lactulose could not be explained by an inactivation of endotoxin. Hypothetically this inhibitory effect of lactulose on the production of tumour necrosis factor may explain the beneficial effect of lactulose as supportive preoperative treatment in jaundiced patients.

1 Pain JA, Cahill CJ, Bailey ME. Perioperative complications in obstructive jaundice: therapeutic considerations. Br F Surg 1985; 72: 942-5.

2 Deventer $\mathrm{v}$ SJH, Cate $\mathrm{t}$ JW, Tytgat GNJ. Intestinal endotoxemia. Gastroenterology 1988; 94: 825-31.

3 Wardle EN, Wright NA. Endotoxin and acute renal failure associated with obstructive jaundice. $\mathrm{Br}$ Med $\mathcal{F} 1970 ; 4$ : associat

4 Wilkinson SP, Moody H, Stamatakis JD, Kakkar VV, Williams R. Endotoxaemia and renal failure in cirrhosis and obstructive jaundice. $\mathrm{BrMed} \mathcal{F}$ 1976; 2: 1415-8.

5 Pain JA, Bailey ME. Measurement of operative plasma endotoxin levels in jaundiced and non-jaundiced patients. Eur Surg Res 1987; 19: 207-16.

6 Corrigan JJ, Kiernat JF. Effect of polymyxin B sulfate on endotoxin activity in a gram-negative septicemia model. Pediatr Res 1979; 13: 48-51.

7 From AHL, Fong JSC, Good RS. Polymyxin B sulfate modification of bacterial endotoxin: effects on the develop-
ment of endotoxin shock in dogs. Infect Immun 1979; 23: $660-4$.

8 Nolan JP, Leibowitz AI. Endotoxin and the liver III. Modification of acute carbon tetrachloride injury by Modification of acute carbon tetrachloride injury by
polymyxin B an antiendotoxin. Gastroenterology 1978; 75: polymyxin
445-9.
9 Ingoldby $\mathrm{CJH}$. The value of polymyxin $\mathrm{B}$ in endotoxaemia due to experimental obstructive jaundice and mesenteric ischaemia. Br F Surg 1980; 67: 565-7.

10 Ingoldby CJ, McPherson GAD, Blumgart LH. Endotoxemia in human obstructive jaundice. Effect of Polymyxin B. Am F Surg 1984; 147: 766-71.

11 Cahill CJ. Prevention of postoperative renal failure in patients with obstructive jaundice - the role of bile salts. Br F Surg 1983; 70: 590-5.

12 Bailey ME. Endotoxin, bile salts and renal function in obstructive jaundice. $\operatorname{Br} \mathcal{F}$ Surg 1976; 63: 774-8.

13 Pain JA, Bailey ME. Experimental and clinical study of lactulose in obstructive jaundice. Br F Surg 1986; 73: 775-8.

14 Liehr H, Englisch G, Rasenack U. Lactulose-A drug with antiendotoxin effect. Hepatogastroenterologv 1980; 27: 35660 .

15 Beutler B, Krochin N, Milsark IW, Luedke C, Cerami A. Control of cachectin (tumor necrosis factor) synthesis: Mechanisms of endotoxin resistance. Science 1986; 232: 977-80.

16 Beutler B, Milsark IW, Cerami A. Passive immunization against cachectin/tumor necrosis factor protects mice from lethal effect of endotoxin. Science $1985 ; 229: 869-71$.

17 Tracey KJ, Fong Y, Hesse DG, et al. Anti-cachectin/TNF monoclonal antibodies prevent septic shock during lethal bacteriaemia. Nature 1987; 330: 662-4.

18 Beutler B, Cerami A. Cachectin: more than a tumor necrosis factor. N Engl f Med 1987; 316: 379-85.

19 Lehman V, Freudenberg MA, Galanos C. Lethal toxicity of lipopolysaccharide and tumor necrosis factor in normal and d-galactosamine-treated mice. F Exp Med 1987; 165: 657d-ga

20 Graziano RF, Fanger MW. Fc-gamma-RI and Fc-gamma-RII on monocytes and granulocytes are cytotoxic trigger molecules for tumor cells. F Immunol 1987; 139: 3536-41.

21 Debets JHM, Linden v.d. CJ, Dieteren IEM, Leeuwenberg JFM, Buurman WA. Fc-receptor cross-linking induces rapid secretion of tumor necrosis factor (cachectin) by human peripheral blood monocytes. F Immunol 1988; 141 : 1197-201.

22 Friberger P, Sörskog L, Nilsson K, Knös M. The use of a quantitative assay in endotoxin testing. Prog Clin Biol Res 1987; 231: 149-69.

23 Olofsson P. Application of a quantitative spectrophotometric endotoxin assay on lymph and plasma from the rat. Eur Surg Res 1986; 18: 112-21.

24 Duff GW, Atkins E. The inhibitory effect of polymyxin B on endotoxin-induced endogenous pyrogen production. endotoxin-induced endogenous pyri
f Immunol Methods 1982; 52: 333-40.

25 Spear GT, Teodorescu M. Enhancement of human MLR by very low concentrations of lipopolysaccharide and blocking
of this enhancement by polymyix B. F Immunol Methods 1984; 73: 321-7.

26 Bircher J, Muller J, Guggenheim P, et al. Treatment of chronic portalsystemic encephalopathy with lactulose. Lancet 1966; i: $890-3$.

27 Vince AJ, Burridge SM. Ammonia production by intestina bacteria: the effects of lactose, lactulose and glucose. $\mathcal{F} \mathrm{Med}$ Microbiol 1980; 13: 177-91.

28 Vince A, Killingley $M$, Wrong OM. Effect of lactulose on ammonia production in a fecal incubation system. Gastroenterology 1978; 74: 544-9.

29 Leeuwen v PAM, Janssen MA, Soeters PB, Jonge d H Metabolic characteristics of villus and crypt cells of conventional and germ-free rats and the influence of Neomycin and tional and germ-free rats and the influend
Lactulose. Eur Surg Res 1985; 17: 77.

30 Huhtanen CN, Parrhish FW, Hicks KB. Inhibition of bacteria by lactulose preparations. Appl Environ Microbiol 1980; 40 $171-3$

31 Delhunty T, Hollander D. A comparison of intestina permeability between humans and three common laborator animals. Comp Biochem Physiol 1987; 86: 565-7.

32 Nathavitharana KA, Lloyd DR, Raafat F, Brown GA McNeish AS. Urinary mannitol: lactulose excretion ratios and jejunal mucosal structure. Arch Dis Child 1988; 63: $1054-9$.

33 Turner MW, Boulton P, Shields JG, et al. Intestinal hypersensitivity reactions in the rat $I$. Uptake of intact protein permeability to sugars and their correlation with mucosal mast-cell activation. Immunology 1988; 63: 119-24.

34 Ziegler TR, Smith RJ, O'Dwyer ST, Demling RH, Wilmor DW. Increased intestinal permeability associated with DW. Increased intestinal permeability associated wits
infection in burn patients. Arch Surg 1988; 123: 1313-9. 